\title{
An Enhanced Resource-Constrained Critical Path Method (eRCPM)
}

\author{
Diana M. Franco Duran ${ }^{1}$ and Jesús M. de la Garza ${ }^{2}$ \\ 1 Virginia Tech, Blacksburg, United States, dianitaf@vt.edu \\ 2 Clemson University, Clemson, United States, jdelaga@clemson.edu
}

\begin{abstract}
The Resource-Constrained Critical Path Method (RCPM) identifies resource-dependent activity relationships (links) when mitigating a resource-supply demand problem. These resource links allow the identification of a continuous critical path and the calculation of correct float values. This paper presents the application of an Enhanced RCPM (eRCPM) in a progressed resource-constrained schedule.

The Enhanced RCPM 1) performs three different serial-based resource-constrained scheduling heuristics (one of those was developed by the authors of this study), 2) keeps and removes specific resource links in a progressed schedule before re-running the algorithm, and 3) selects a default schedule after evaluating some schedule characteristics. Additionally, an Enhanced RCPM system was developed and integrated with Primavera P6.

This system imports and reads data from a P6 project; performs the Enhanced RCPM; updates the P6 file; and puts the project back into the Primavera P6 database. From this updated schedule, users can get: 1 ) correct early and late CPM dates; 2) correct float values; 3 ) a continuous critical path; 4) the resources links that were added into the schedule, and 4) the amount of phantom float (float that does not exist) each activity had before adding the resource links into the schedule. The development of the eRCPM computerized system allows the identification of a continuous critical path, practically, in P6 resourceconstrained schedules.
\end{abstract}

(c) 2020 The Authors. Published by Budapest University of Technology and Economics \& Diamond Congress Ltd Peer-review under responsibility of the Scientific Committee of the Creative Construction Conference 2020.

Keywords: phantom float, Primavera P6, resource overallocation, resource-constrained scheduling, resource-depend activity relationships

\section{Introduction}

The baseline schedule is frequently used to track project performance [1]. Resources, as a key component of schedules, must be also monitored to prevent or mitigate any extension on the Project Completion Time (PCT) because of resource availability. When the resource demand exceeds the supply, activities must be delayed until resources become available.

Due to the projects' size and complexity, schedulers use scheduling software such as Primavera P6 or Microsoft Project to fix the resource conflicts of a schedule. Even though the software solves the overallocation problem applying Resource-Constrained Scheduling (RCS) methods, the results show incorrect total float values and a broken critical path. This happens because RCS calculations suggest that activities have float but much of this float does not exist - hence the named Phantom Float [2]; [3].

Even though the Resource-Constrained Critical Path Method (RCPM) correctly calculates the floats of activities and identifies a continuous critical path in resource-constrained schedules, some shortcomings 
must be addressed to enhance its capability and make it more practical for real construction projects [3], [4]. This study tackles some of the flaws of the RCPM and illustrates the application of the Enhanced RCPM (eRCPM) with a case study.

\section{RCPM shortcomings}

This section briefly describes some of the flaws of the RCPM. A detailed literature review identifying the shortcomings of RCS-related algorithms can be found in [4].

\subsection{Priority rules}

Heuristics are problem-dependent, so they are likely to be better in some situations than in others. Some priority rules may work well for a project but may not work well when applied to a different project [5]. Even if the PCT obtained by two or more heuristics is the same, the sequence of the activities may be different [6].

The RCPM applies the Late Start (LS) heuristic to mitigate a resource-supply demand problem. Since each heuristic works differently and produces different schedule outcomes, the eRCPM incorporates 1) two additional heuristics (ES and Enhanced LF) and 2) a criterion to evaluate the resulting schedules and to select one as a default.

\subsection{Removal of resource links}

The RCPM was developed to solve the issue of a broken critical path in a resource-constrained schedule. Hence, Kim and de la Garza (2003) did not explore the application of the RCPM for control purposes further i.e., the use of resource links in a progressed schedule.

The updates on a baseline/progressed schedule could change the priority order identified by a heuristic to schedule the activities when an over-allocation problem exists. When re-applying the RCPM, the resource links identified before updating the project may no longer be required and/or new resource links can be identified because of the changes in the schedule. Some of the existing resource links should be removed from the schedule because they were identified based on previous and different conditions. If these links are kept, they constrain the schedule.

The RCPM removes all existing resource links before re-running the algorithm in a progressed schedule [3]. The eRCPM removes only the resource links located right to the data date each time a project is updated, and the algorithm is re-applied. The eRCPM keeps the resource links located left to the data date because the project was already executed based on these activities' configurations.

\subsection{Selecting resource-driving activities}

One issue that arises when identifying activity resource relationships is having different possible links configurations between activities [7], [8]. This occurs when having many current activities with many predecessors (see Fig. 1). The difference between the different schedules that can be generated is not only the number of resource links created but also the number of critical activities.
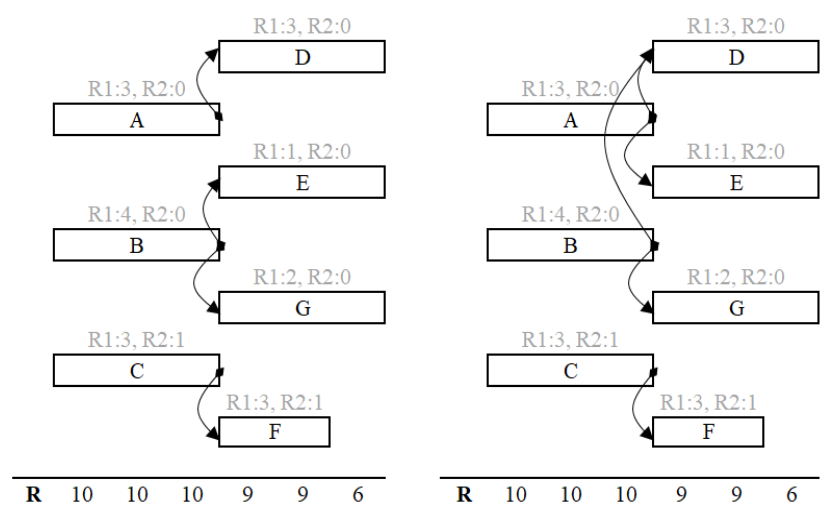

Fig. 1. Multiple Schedule Alternatives. Example taken from [8]. 
According to Nisar, Yamamoto, and Suzuki (2013), the resource dependences should be created in a way the total number of relationships is minimized without violating the resource constraints. The goal is to not increase the complexity of a network with a high number of resource relationships.

The RCPM does not incorporate any criteria to identify resource-driving activities. Instead, the RCPM creates all possible resource links configurations between the activities under consideration [3]. Given the influence on extending a PCT, the eRCPM considers the resources and duration of the activities as the main criteria to select a resource-driving activity.

\subsection{RCPM prototype system}

The RCPM prototype system developed by Kim and de la Garza in 2003 for Project Planner (P3) does not work for Primavera P6 because P6 is built on a different platform than P3. At present, there is a lack of practical mechanisms to identify resource relationships in P6 project schedules. Hence, the eRCPM was integrated with Primavera P6. The eRCPM system reads information from a P6 project, performs the necessary eRCPM procedures, and updates the P6 project with the corresponding resource relationships.

\section{Enhanced Resource-Constrained Critical Method (eRCPM)}

Figure 2 shows a comparison of the RCPM and eRCPM. This section explains each of the steps of the eRCPM (see Fig. 2b).

a

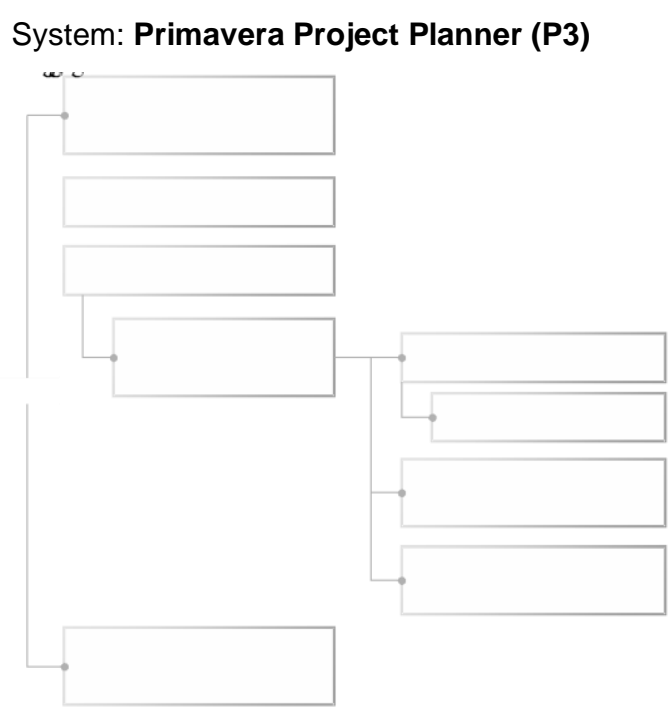

b

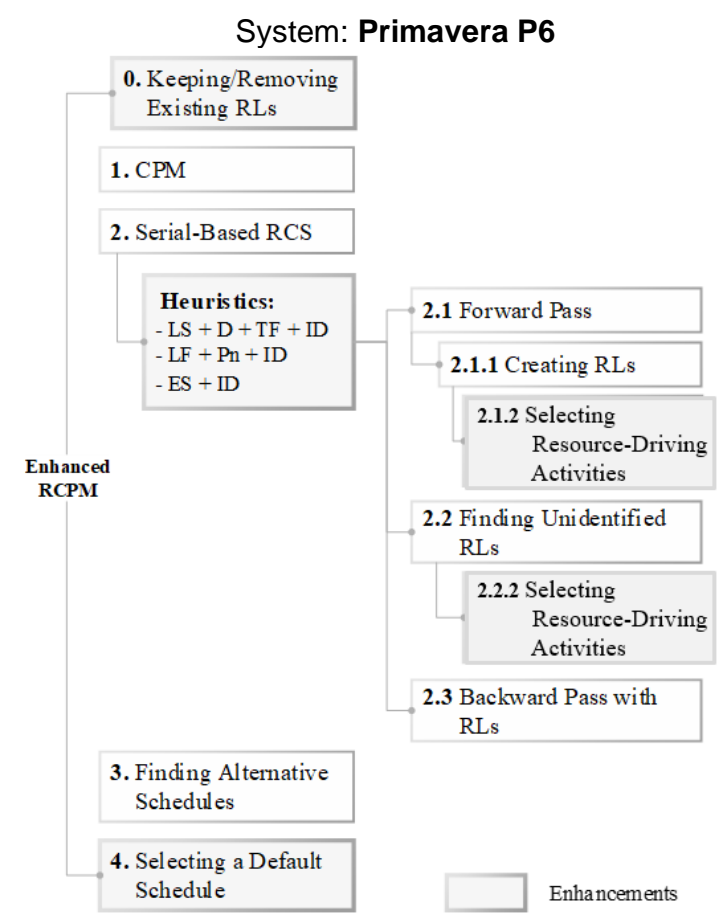

Fig. 2. Outline: (a) RCPM; (b) Fig. 2. Enhanced RCPM (eRCPM).

\subsection{Step 0 - Keeping/removing resource links}

In a progressed schedule with resource relationships, the eRCPM checks the status of each activity to determine whether a Resource Link (RL) should be removed from or kept on the schedule. The RLs are kept in the schedule if 1) both activities (predecessor and successor) are already complete and 2) the predecessor activity is complete, and the successor activity is in progress. Otherwise, the RLs are removed from the schedule if 1 ) the two activities (predecessor and successor) have not started yet and, 2) the predecessor activity is complete, but the successor activity has not started (see Fig. 3). 


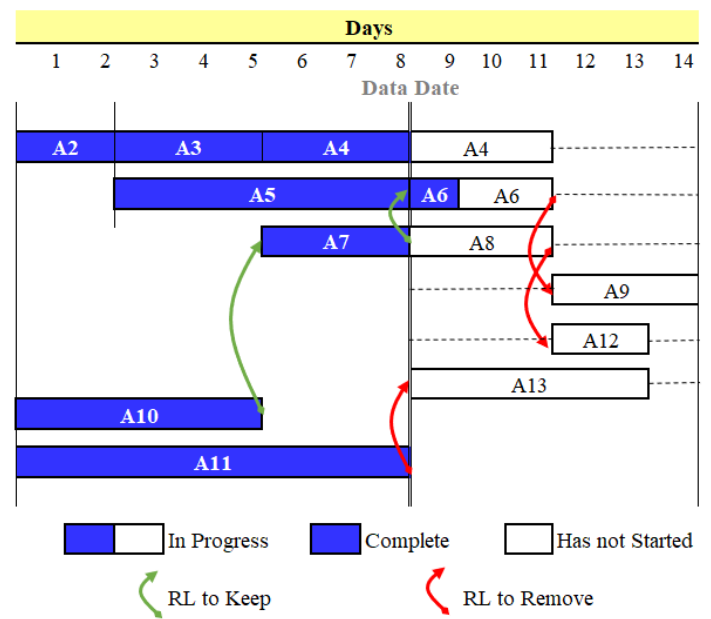

Fig. 3. Keeping and Removing Existing Resource Links before re-applying the eRCPM.

\subsection{Step 1- Critical Path Method (CPM)}

The eRCPM performs the CPM to find the early and late dates, and float values of each activity. If there is a resource overallocation problem, the eRCPM mitigates the resource-supply demand problem by applying resource-constrained heuristics.

\subsection{Step 2 - Serial-based RCS heuristics with resource links}

The eRCPM performs three different heuristics to mitigate a resource supply-demand problem: 1) Late Start (LS), 2) Enhanced Late Finish (LF), and 3) Early Start (ES). These three heuristics were incorporated into the algorithm because they produce better results in terms of extending the project duration than other existing priority rules [8]. The heuristics are performed under a serial approach, e.g., activities are sorted as a single group and then scheduled one at a time.

- In the Late Start heuristic, the priority is given to activities with the earliest values of Late Start (LS). If there is a tie with respect to the $L S$, the priority is given to the activity with the least duration (D). If the tie persists, the priority is given to the activity with the least total float (TF). If the tie persists, the priority is given to the activity with the smallest activity number (ID).

- In the Enhanced LF heuristic, the priority is given to activities with the earliest values of Late Finish (LF). If there is a tie with respect to the LF, the priority is given to the activity with the lowest Priority Number (Pn). The Priority Number, which is a tiebreaker that can be incorporated with any rule, is calculated based on the duration (Di) and total float (TFi) of each activity (see Equation 1). When an activity is critical ( $\mathrm{TFi}=0$ ), the TFi is assumed to be equal to 0.95 . For an activity in progress, the remaining duration of the activity is used to calculate the Pn. If there is a tie with respect to the Pn, the tie is broken by the smallest activity number (ID). The formal definition of this new heuristic can be found in [9].

$$
\mathrm{Pn}=\mathrm{Di} / \mathrm{TFi}
$$

- In the Earliest Start heuristic, the priority is given to activities with the earliest values of Early Start (ES). If there is a tie with respect to the ES, the tie is broken by the smallest activity number (ID). 


\subsubsection{Step 2.1: Forward pass}

Step 2.1.1 - Creating Resource Links: During the performance of any of the three RCS heuristics mentioned above, if there are not enough resources to execute an activity, the activity is delayed until resources become available. The resources causing the current activity delay are released from other activity completion [3]. Like the RCPM, the eRCPM creates a resource link (relationship) between the postponed activity (successor) and the preceding activity that shares the same resources (resource-driving activity).

Step 2.1.2 - Selecting resource-driving activities: The eRCPM considers three different cases to identify the "resource-driving activity" for the delayed task when having multiple alternatives.

\section{Case I - One type of resource}

When having one type of resource, the eRCPM selects as a resource-driving activity, the activity with the highest number of resources. If there is a tie with respect to the number of resources, the activity with the longest duration is selected. If the tie persists, the activity with the smallest activity ID is selected as a resource-driving.

For example, in Fig. 4, Activity A7 is delayed because of resource unavailability during days 1 to 8 (ten resources would be needed but only eight are available). Either A4 or A11 are the potential resource-driving activity of A7. For this scenario, the traditional RCPM creates two resource links, one between A4 and A7, and another between $\mathrm{A} 11$ and A7. The eRCPM creates only one link between A4 and A7 because A4 requires a higher number of resources than $A 7(R=2$ vs $R=1)$.

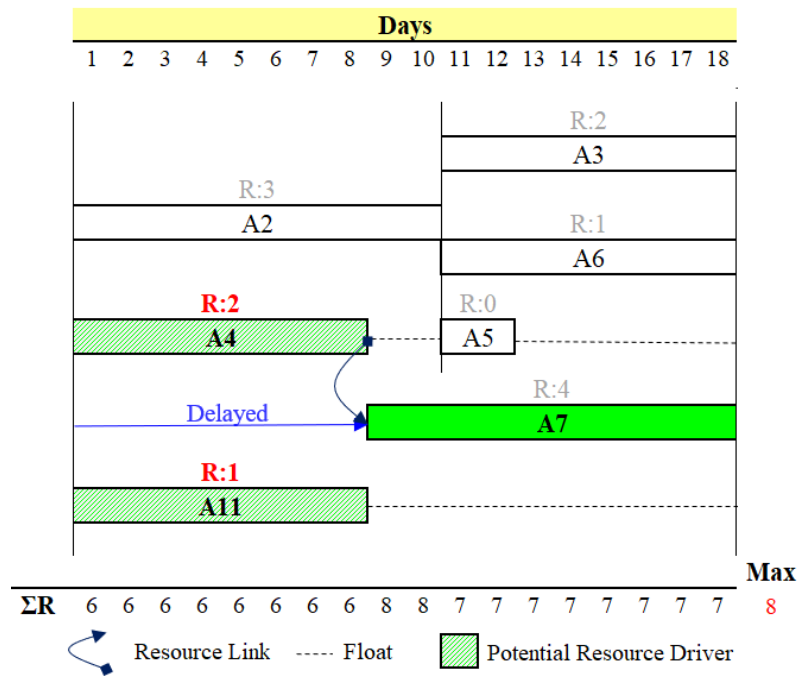

Fig. 4. Example of Case I: One Type of Resources.

\section{Case II - Two types of resources and 1 conflicting resource}

When having two types of resources and only one conflicting resource type, the eRCPM selects as a resource-driving activity, the activity with the highest number of conflicting resources. If there is a tie with respect to the higher number of resources, the activity with the longest duration is selected. If the tie persists, the activity with the highest number of the other type of resource is selected. If the tie persists, the activity with the smallest activity ID is selected.

In Fig. 5, Activity A11 was delayed because of the resource unavailability of R1. Activities A2, A4, and A10 are the potential resource-driving activities of A11. The traditional RCPM creates three RLs, one between A11 and $A 2$; other between $A 11$ and $A 4$; and another between $A 11$ and A10. The eRCPM creates only a link between $A 10$ and $A 11$. In this case, although $A 10$ and $A 4$ have the same higher number of the conflicting resource $(R 1=3)$ and the same duration $(D=7$ Days), $A 10$ requires more resources type two $(R 2=2)$ than A4 $(\mathrm{R} 2=0)$. 


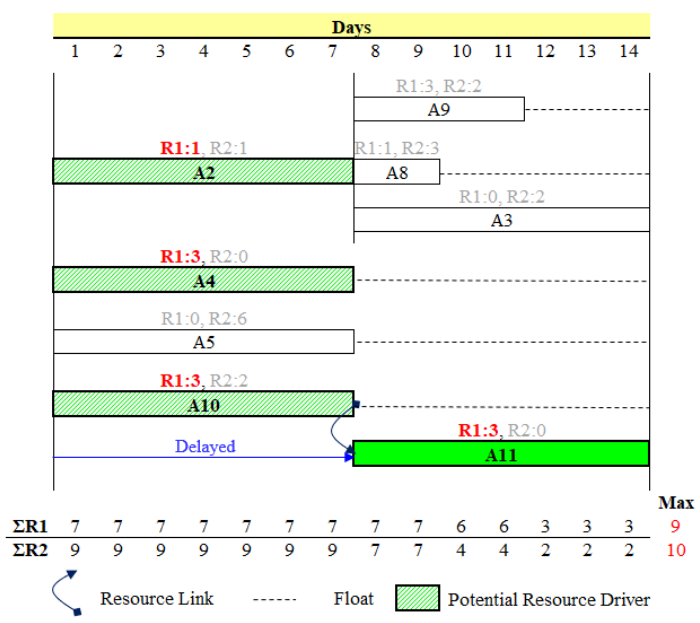

Fig. 5. Example Case II: Two Types of Resources and One Conflicting Resource.

\section{Case III - Two or more conflict resources}

When having two or more types of resources and several conflicting resource types, the eRCPM algorithm selects as a resource-driving activity, the activity with the highest average number of conflicting resources. If there is a tie with respect to the average number of conflicting resources, the activity with the longest duration is selected. If the tie persists, the activity with the smallest activity ID is selected as a resourcedriving.

In Fig. 6, Activity A11 was delayed because of the resource unavailability of R2 and R3 (conflicting resource types). Activities $A 3, A 7$, and $A 8$ are the potential resource-driving activities of $A 11$. The traditional RCPM creates three RLs, one between $A 3$ and $A 11$, other between $A 7$ and $A 11$, and another between $A 8$ and $A 11$. The eRCPM creates only one link between $A 8$ and $A 11$. In this case, $A 8$ has a higher average number of the two conflicting resources (Average $A 8=3$ ) than the other two activities (Average $A 3=2.5$; Average $A 7=2$ ).

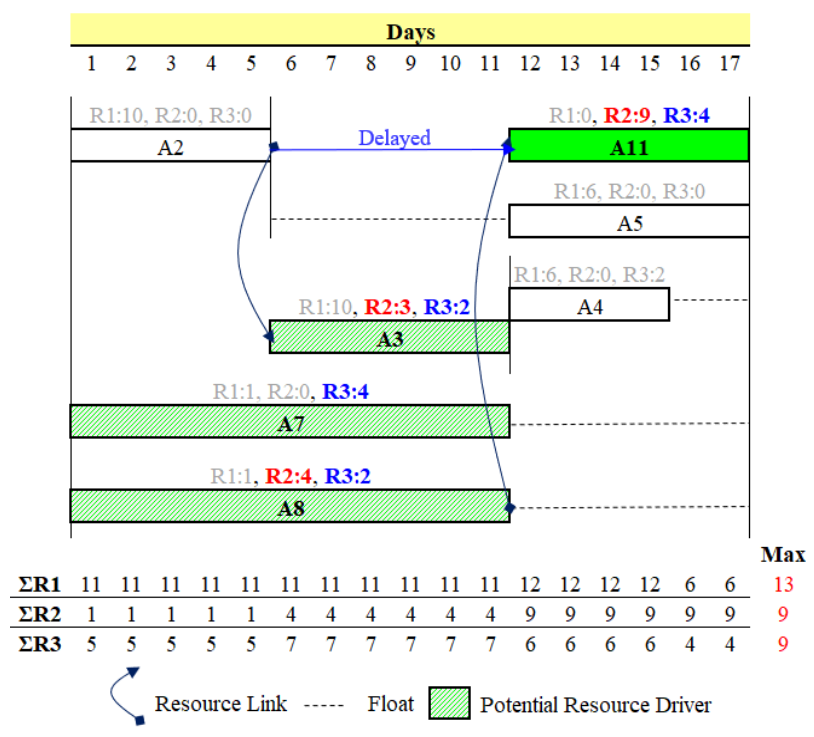

Fig. 6. Example Case III: Two or more Conflict Resources.

\subsubsection{Step 2.2 Finding unidentified resource links}

Like RCPM, before performing the backward pass, the eRCPM checks if non-critical activities (non-zero total float) can fully use the float (TF) or if there is any resource constraint for the float period [3]. If so, an additional resource link is created between the conflicting activities considering the three cases described above when having multiple possible resource-driving activities.

In Fig. 7, when checking for unidentified RLs, Activity A4, which has "apparently" seven days of float (Days 8 to 14), cannot be delayed because otherwise, an over-allocation arises with respect to R1 (11 resources will 
be needed and there are only nine available). Activities $A 8, A 9$, and $A 11$ are the potential resource-driving activities of $A 4$. The traditional RCPM creates three RLs, one between $A 4$ and $A 8$, other between A4 and A9, and another between $A 4$ and $A 11$. The eRCPM creates only a link between $A 4$ and $A 11$. In this case, although $A 11$ and $A 9$ have the same higher number of resources, the duration of $A 11$ ( $D=7$ Days) is longer than $A 9$ $(\mathrm{D}=4$ Days $)$.

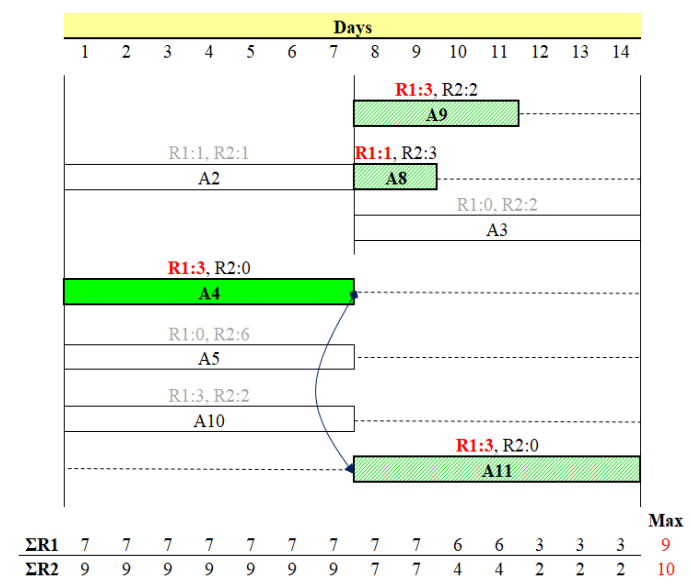

Fig. 7. Example: Identification of additional Resource Links.

3.3.3. Step 2.3 - Backward pass: Once all resource links are identified, the eRCPM performs the CPM backward pass considering both the technological and resource relationships. By considering both types of relationships, a continuous critical path can be identified in a resource-constrained schedule.

\subsection{Step 3 - Alternate schedule}

Like RCPM, the eRCPM finds alternative schedules by looking for activities that can be scheduled during a different period without breaching all the relationships.

\subsection{Step 4 - Selecting a schedule}

Since the eRCPM performs three different RCS heuristics (LS, Enhanced LF, and ES), the algorithm selects as a default schedule the one with the shortest duration. If there is a tie with respect to the Project Completion Time (PCT), the schedule with the smallest resource moment value (Mx) is selected.

The Minimum Moment ( $\mathrm{Mx}$ ) was chosen as a criterion to select a resulting resource-constrained schedule because it is a good measure of resource utilization. A lower value indicates a better resource allocation, e.g., a resource profile closer to a rectangular shape. The moment of the daily resource demands about the horizontal axis of a project's resource histogram (Mx) is calculated as shown in Equation 2 [10]. Where yi represents the daily resource utilization. When having multiple types of resources in a schedule, $\mathrm{Mx}$ is calculated for each resource profile and then compared with the values of the other schedules. The schedule with the highest number of resource profiles with the lowest Mx is selected as a default schedule. The Mx is calculated after the data date for progressed schedules.

$$
\mathrm{Mx}=12 \mathrm{i}=1 \text { nyi2 }
$$

For example, the two schedules show in Fig. $8 \mathrm{a}$ and $8 \mathrm{~b}$ (LS-based and ES-based respectively) have the same PCT (19 days) but different resource profiles. Since the Mx of the resource profile of the LS-based schedule is lower (197.0) than the that of the ES-based schedule (200), the eRCPM selects as default the LS-based schedule. The resource profile of this schedule is closer to a rectangular shape than that of the ES-based schedule and therefore, it has better resource allocation. 
a

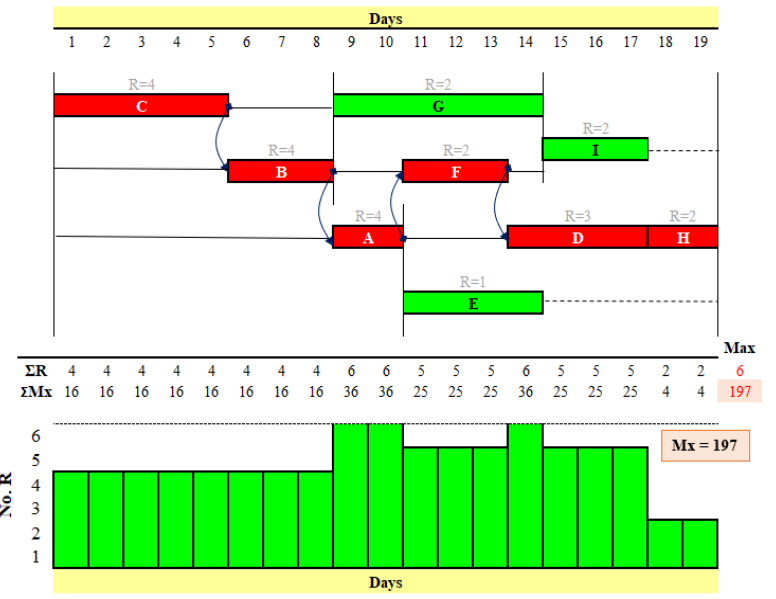

b
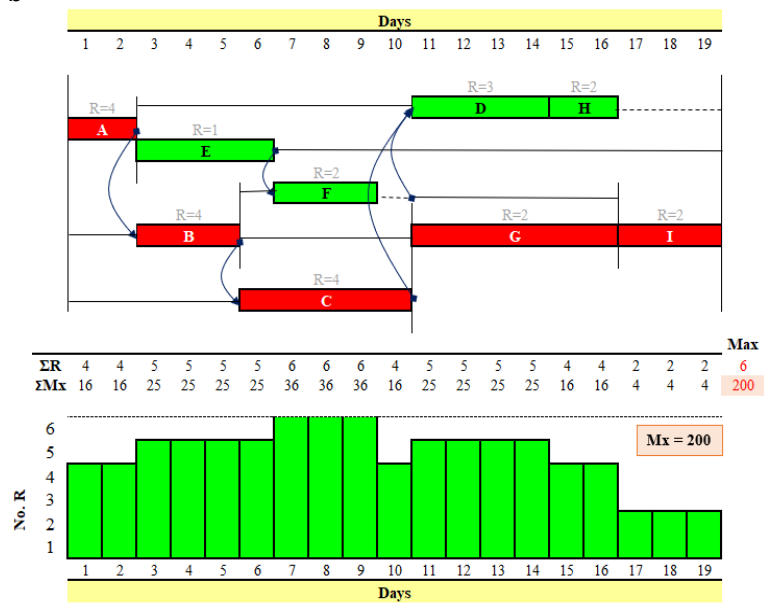

Fig. 8. Resource Profiles: Calculation of Mx: (a) LS-based Schedule; (b) ES-based Schedule.

If there is a tie with respect to the $M x$, the schedule with the lowest number of critical activities $(T F=0)$ is selected as a default. If the tie persists, the schedule with the highest average of free float is selected as a default. The float values (TF and FF) were selected as parameters to select a resource-constrained schedule because 1) having a schedule with less critical activities decrease the probability of delaying the project completion time, and 2) having a schedule with a higher average time of free float gives more flexibility for delaying some activities without affecting the start time of the successor activities.

Finally, if there is still a tie between the three resulting schedules (LS, Enhanced LF, and ES based), the eRCPM selects the LS-based schedule as a default. If the tie is between the Enhanced LF and ES-based schedules, the eRCPM selects as the default the Enhanced LF-based schedule.

\section{Enhanced RCPM (eRCPM) system}

The eRCPM system was developed and integrated with Primavera P6 using the Primavera P6's Application Program Interface (API). The eRCPM prototype system handles smart relationships, multiple calendars, holydays and exceptions, multiple types of resources, and progressed schedules. The system 1) exports a P6 project in an XML format, 2) reads the project information from the XML file, 3) performs the Enhanced $R C P M, 4)$ updates the $X M L$ file by adding the identified resource relationships, and 5) imports the $X M L$ file again into P6. Then, the updated schedule appears in the user's P6 database (see Fig. 9).

Since the updated P6 file has already the resource relationships (RLs) incorporated into the schedule, the user should only re-run CPM in P6 (schedule -F9) to obtain the early/late dates and float values. Primavera's users can identify the resources links that were added to the schedule adding a user-defined field created by the eRCPM system called "RL Successors". The user-defined fields are custom fields that P6 users can create to track specific project information. The eRCPM system also creates another user-defined field called "PF", which shows the phantom float (non-existent float) each activity had before adding the resource links into the schedule. Finally, if after comparing all three resulting schedules (LS, Enhanced LF, and ES based), the program selects as default the LF-based schedule, P6 users can add another user-defined field called "PN". This column shows the Priority Number used for each activity when applying the Enhanced LF heuristic. 


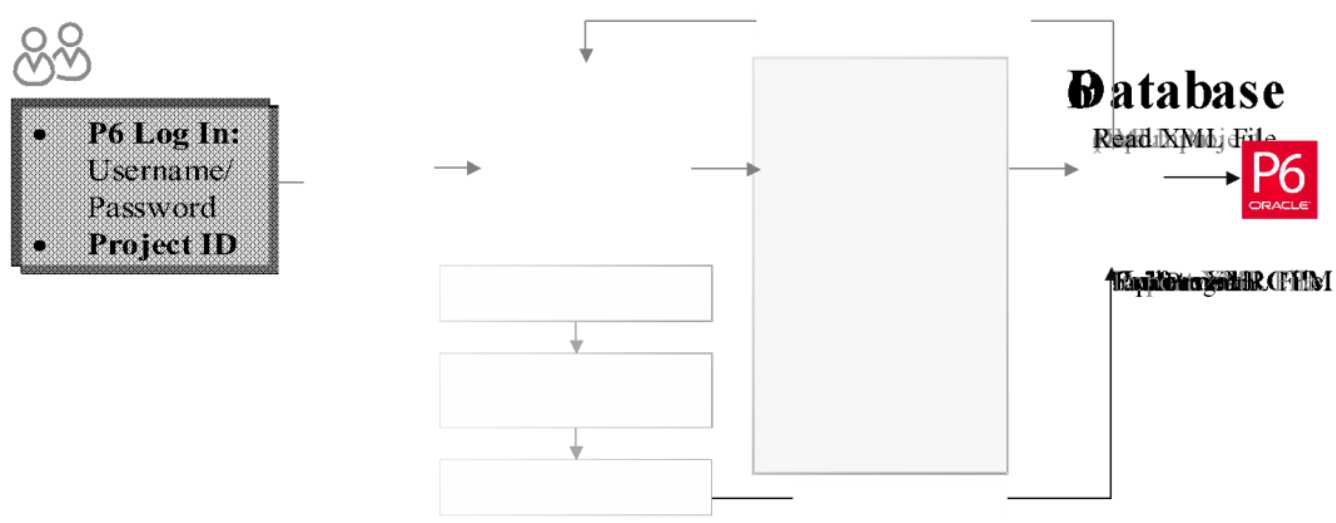

Fig. 9. Enhanced RCPM System.

\section{5. eRCPM application}

This section shows the application of the eRCPM to a progressed resource-constrained schedule. The eRCPM was performed by 1) hand and the resulting schedule was drawn in a fenced bar char and 2) using the eRCPM System.

This case study schedule consists of nine activities with two types of precedence relationships (FS and SS), two types of resources (R1 and R2), and two different calendars. Calendar 1 has seven workdays per week and Calendar 2 has five workdays per week. Both calendars have two days of exceptions (non-working days), October $23^{\text {rd }}$ and November $1^{\text {st. }}$ The maximum number of resources available per day for R1 is six and $\mathrm{R} 2$ is seven. Fig. 10 shows the network of the schedule.

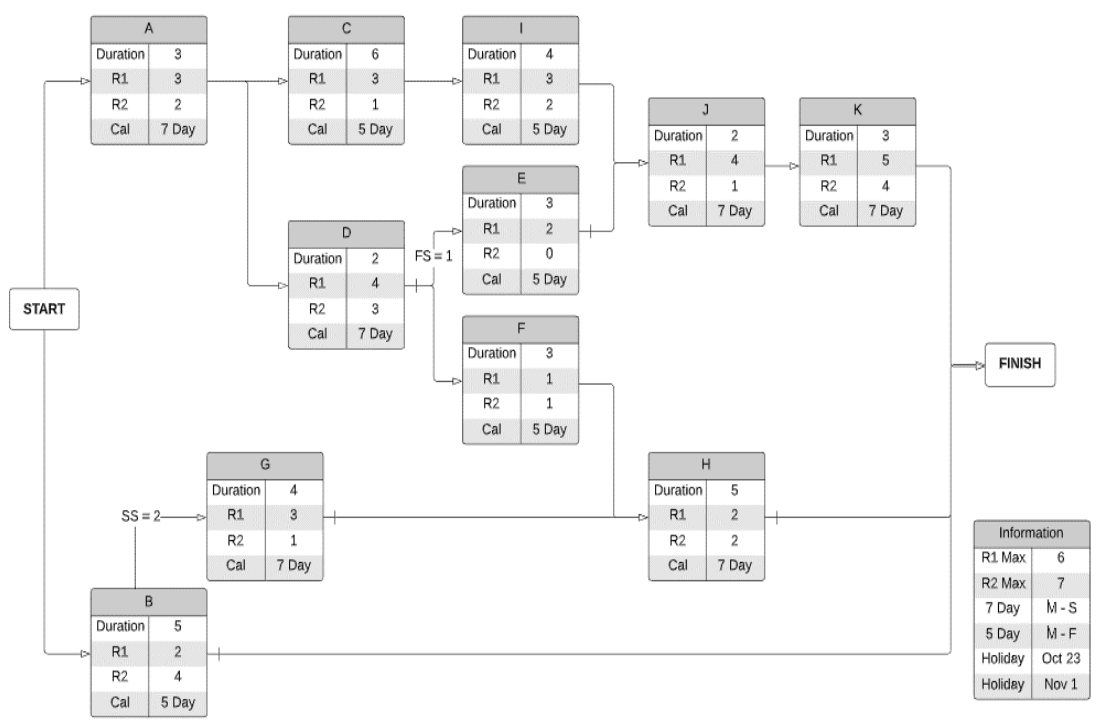

Fig. 10. Case Study: Network.

Fig. 11 shows the CPM fenced bar chart of the project. The CPM results indicated a Project Completion Time (PCT) of 23 days with activities $A, C, I, J$, and $K$ as critical. As shown in Fig. 11, there is an over-allocation problem for R1 during days 9 to 11 and for R2 during days 10 to 11. The eRCPM was applied to mitigate this supply-demand problem. 


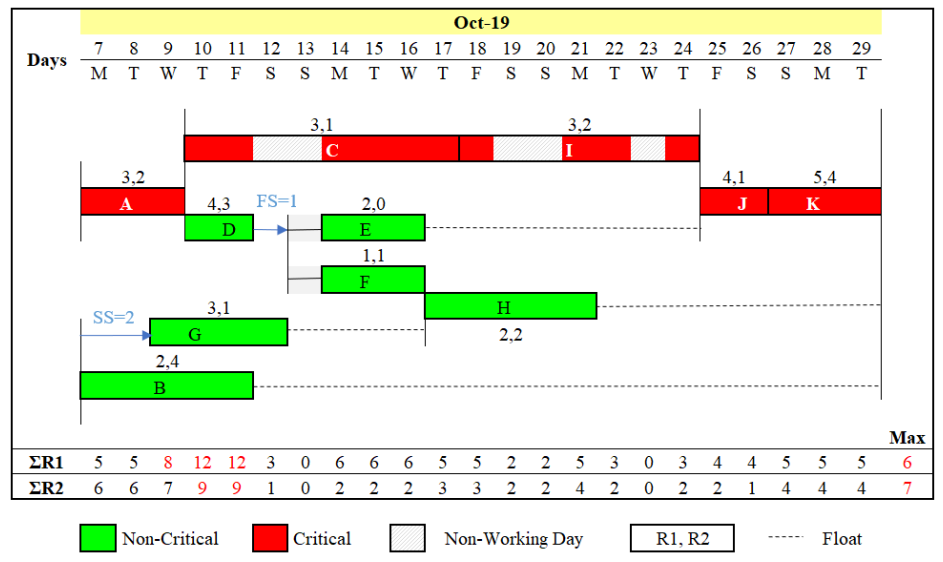

Fig. 11. CPM Fenced Bar Chart.

After applying the three heuristics, the eRCPM system selected the LF-based schedule as default. As shown in Fig. 12, after solving the resource overallocation problem, the PCT was extended by one day with activities $B, D, E, G, H$, and $K$ as critical. Additionally, five resource links were incorporated in the schedule (B-D, C-G, E-G, F-G, and, H-K). This schedule (Fig. 12) was used as a baseline to update the project.

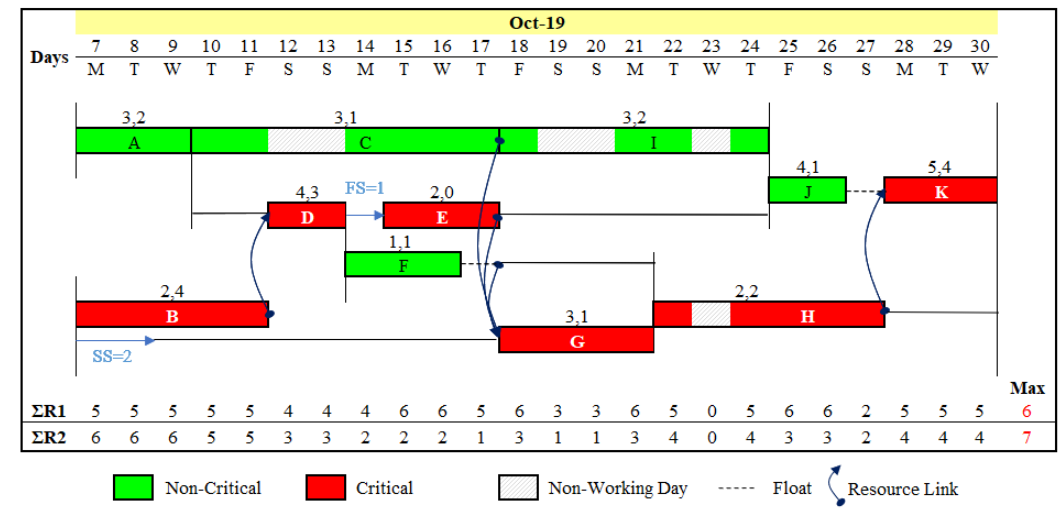

Fig. 12. eRCPM Fenced Bar Chart (LF-based).

The baseline schedule shown in Fig. 12 was updated at the end of week 1 (Oct 13, 2019). Activities A, B, and $D$ have been complete and activity $C$ is still in progress (see Fig. 13). Since this is a progressed schedule, the resource link located left to data date between activities $B$ and $D$ should be kept in the schedule because both activities are complete and the RLs between activities $C-G, E-G, F-G$, and $H-K$ should be removed from the schedule because these activities have not started yet.

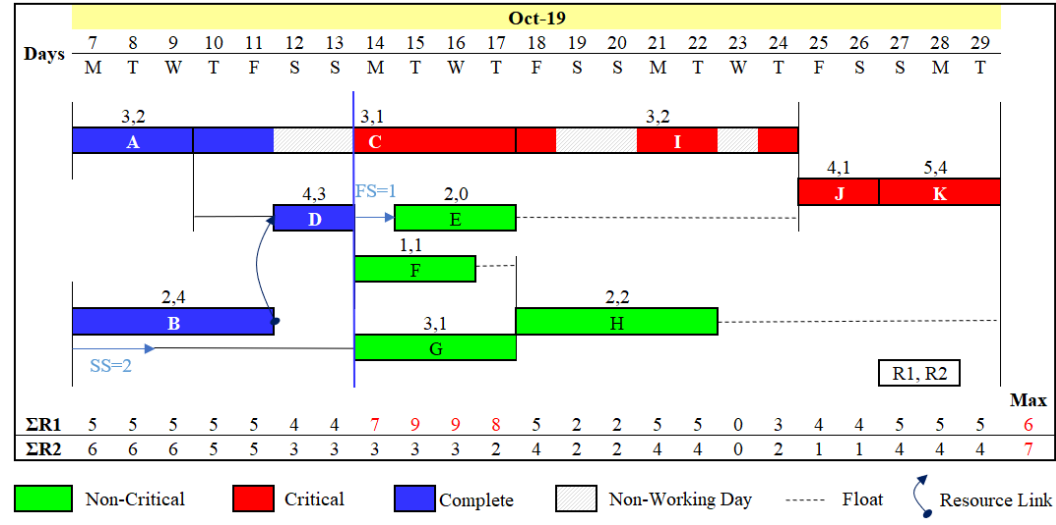

Fig. 13. Fenced Bar Chart: Schedule Updates.

Before re-running the eRCPM, the program identifies and displays the resource links that are kept in and removed from the schedule based on the data date of the project and the status of each activity. Fig. 14 shows the kept and removed RLs identified by the eRCPM system for the case study. With this activity configuration, the eRCPM was re-applied. 


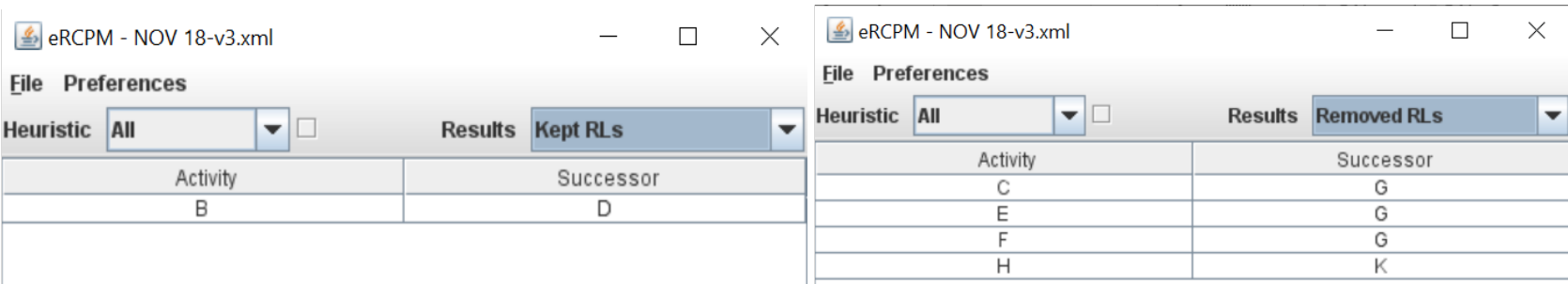

Fig. 14. Resource Links (RLs): (a) Kept (b) Removed, before re-running the eRCPM.

The CPM results indicate a project duration of 23 days (PCT: Oct 27, 2019) with activities C, I, J, and K as critical after the data date. For a progressed schedule, when an activity is complete, the program displays the activity duration as zero (see Fig 15). Additionally, there is an over-allocation problem during days 14 to 17 for R1 (see Fig. 13).

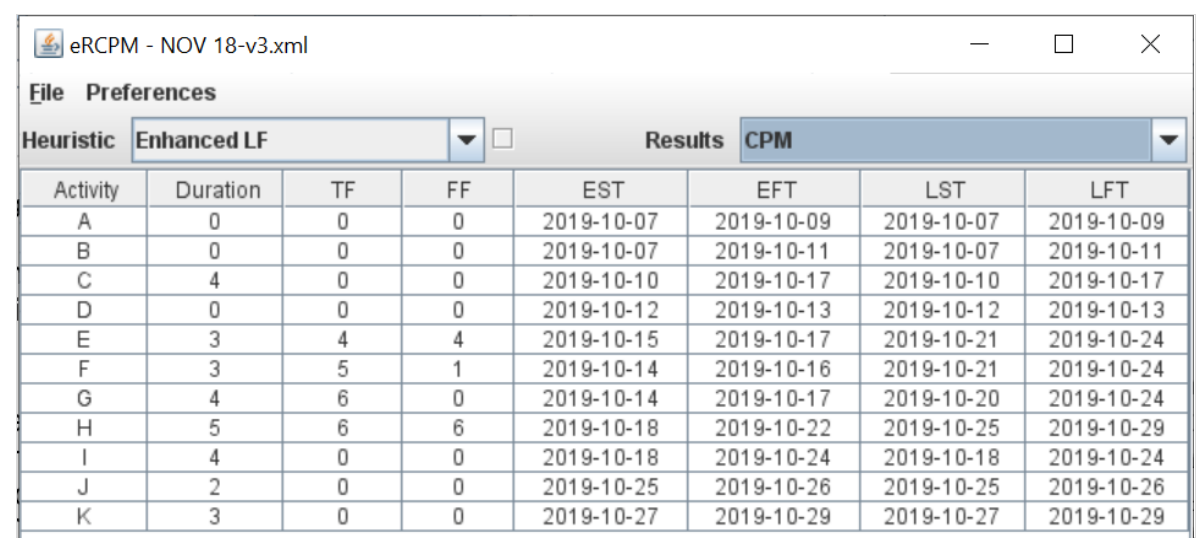

Fig. 15. eRCPM System: CPM Results.

After applying the three heuristics (LS, Enhanced LF, and ES), the eRCPM system selected the Enhanced LFbased schedule as default (recommended) and updated the P6 project based on this heuristic output. Fig. 16 shows the results of the comparison performed by the eRCPM system when selecting the default schedule. Even though the Enhanced LF and LS-based schedules have the same finish date, the Enhanced LF-based schedule was selected as default because it has lower values of Mx for the two types of resources $(\mathrm{Mx} 1=189 ; \mathrm{Mx} 2=64.5)$. Due to the sequence of the activities, the resource allocation of the Enhanced LFbased schedule is better than that of the LS-based schedule.

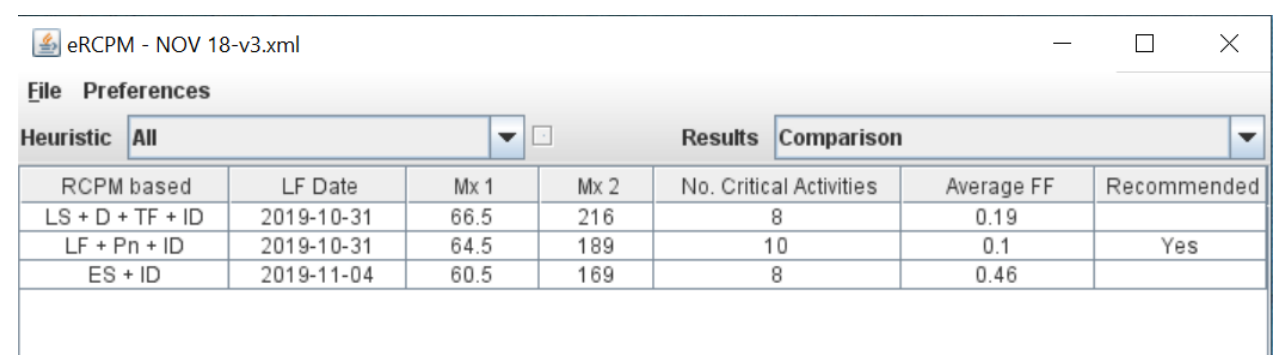

Fig. 16. eRCPM System: Comparison of schedules.

Fig. 17 shows the resulting Enhanced LF-based schedule drawn in a fenced bar chart after re-running the eRCPM in the progressed schedule. The PCT was extended by two days (from 23 days to 25 days) after mitigating the resource supply-demand problem with the Enhanced LF heuristic (from Oct 29, 2019 to Oct 31, 2019). Five RLs were identified and activities C, E, H, and K are critical. 


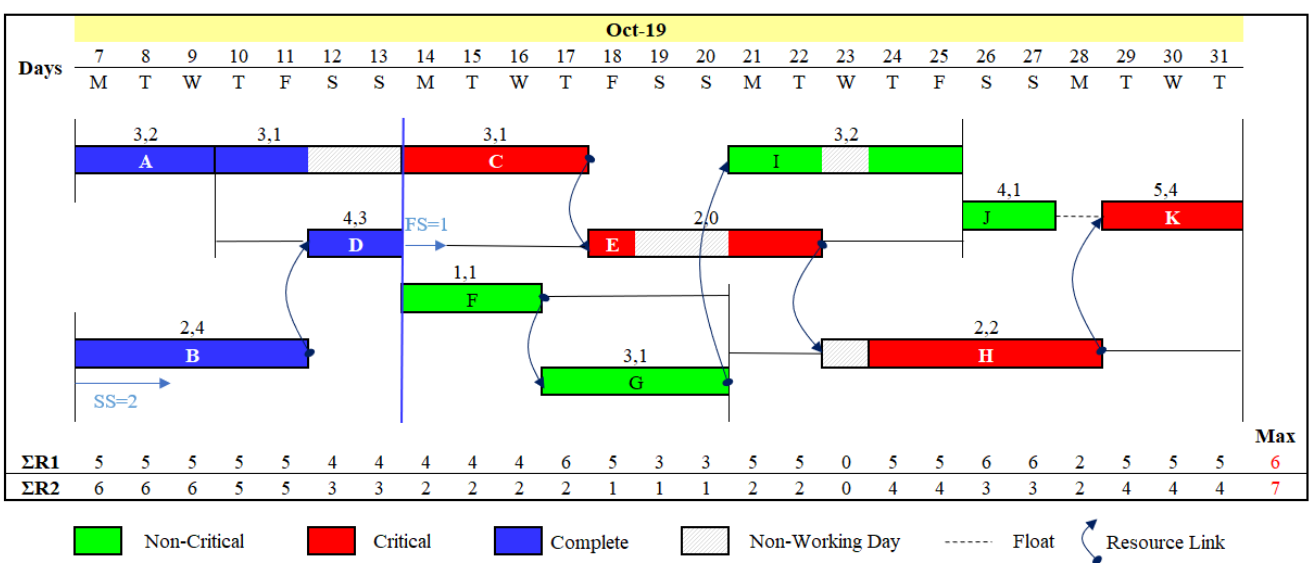

Fig. 17. eRCPM (Enhanced Late Finish Sort): Fenced Bar Chart.

The LS and Enhanced LF-based schedules have the same finish date (Oct 31, 2019) but the sequence of the activities differs and thus, the RLs, and critical path. In the LS-based schedule, activities G, E, F, H, and K are critical. Worthy of note, after the data date, the Enhanced LF-based schedule has a fewer number of critical activities than the LS-based schedule (4 vs 5 ) but a higher number of RLs (5 vs 4 ). Otherwise, the PCT was extended by six days (from 23 days to 29 days) after mitigating the resource supply-demand problem with the ES heuristic (PCT: Nov 4, 2019). In this schedule, activities C, E, I, J, and K are critical.

Fig. 18 shows the output of the program after performing the Enhanced LF. Before adding the resource links into the schedule, five activities had Phantom Float (PF) (C, E, F, G, and H). Since the sequence of the activities changed, the RLs identified after re-applying the eRCPM are different from the ones the schedule had before the update and which were removed before re-running the method (see Fig. 19).

\begin{tabular}{|c|c|c|c|c|c|c|c|c|c|}
\hline \multicolumn{7}{|c|}{ eRCPM - NOV 18-v3.xml } & & & \\
\hline \multicolumn{10}{|c|}{ File Preferences } \\
\hline Heuristic & hanced LF & & & $\nabla \square$ & Results & RCPM & & & $\mathbf{v}$ \\
\hline Activity & Duration & TF & $\mathrm{FF}$ & PF & EST & EFT & LST & LFT & \\
\hline A & 0 & 0 & 0 & 0 & 2019-10-07 & 2019-10-09 & 2019-10-07 & 2019-10-09 & \\
\hline B & 0 & 0 & 0 & 0 & 2019-10-07 & 2019-10-11 & 2019-10-07 & 2019-10-11 & \\
\hline C & 4 & 0 & 0 & 1 & 2019-10-10 & 2019-10-17 & 2019-10-10 & 2019-10-17 & \\
\hline D & 0 & 0 & 0 & 0 & $2019-10-12$ & 2019-10-13 & 2019-10-12 & 2019-10-13 & \\
\hline$E$ & 3 & 0 & 0 & 2 & 2019-10-18 & $2019-10-22$ & 2019-10-18 & $2019-10-22$ & \\
\hline $\mathrm{F}$ & 3 & 0 & 0 & 6 & 2019-10-14 & 2019-10-16 & 2019-10-14 & $2019-10-16$ & \\
\hline G & 4 & 0 & 0 & 5 & 2019-10-17 & 2019-10-20 & 2019-10-17 & 2019-10-20 & \\
\hline $\mathrm{H}$ & 5 & 0 & 0 & 3 & 2019-10-24 & 2019-10-28 & 2019-10-24 & $2019-10-28$ & \\
\hline 1 & 4 & 0 & 0 & 0 & 2019-10-21 & $2019-10-25$ & 2019-10-21 & $2019-10-25$ & \\
\hline $\mathrm{J}$ & 2 & 1 & 1 & 0 & $2019-10-26$ & 2019-10-27 & 2019-10-27 & $2019-10-28$ & \\
\hline $\mathrm{K}$ & 3 & 0 & 0 & 0 & 2019-10-29 & 2019-10-31 & 2019-10-29 & 2019-10-31 & \\
\hline
\end{tabular}

Fig. 18. eRCPM System: RCPM Results of the Enhanced LF-based Schedule.

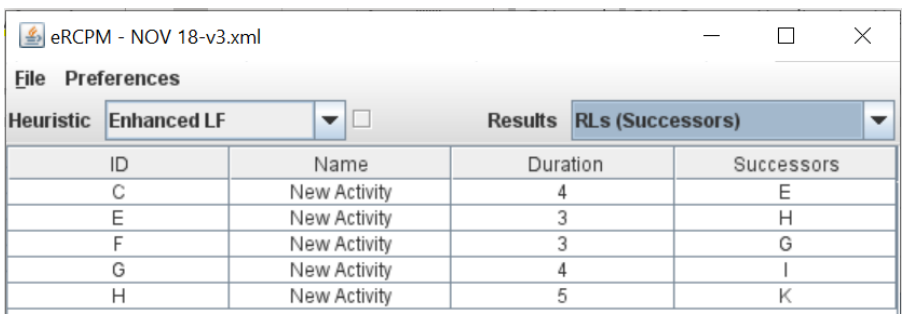

Fig. 19. eRCPM System: Resource Links of the Enhanced LF-based Schedule.

Once the user opens the file and runs the project, P6 updates the early and late CPM dates (EST, EFT, LST, and LFT), as well as, the float values (FF, TF) of each activity. As a result, a continuous critical path can be identified in the P6 resource-constrained schedule. The resulting values displayed by P6 match with the ones obtained by the eRCPM system (see Fig. 17 and 18).

Fig. 20 shows the updated project in P6. The eRCPM system creates resource links in P6 as Finish-to-Start relationships without lag $(\mathrm{FS}=0)$. These new relationships can be identified in a P6 Project by adding the 
user-defined column called "RL Successors". This column indicates the successor resource-driving activity of the activity being considered. For example, in Fig. 20, a new link between activity $C$ and $E$ was added to the schedule (Activity I was already a successor (technological relationship) of activity A before performing eRCPM).

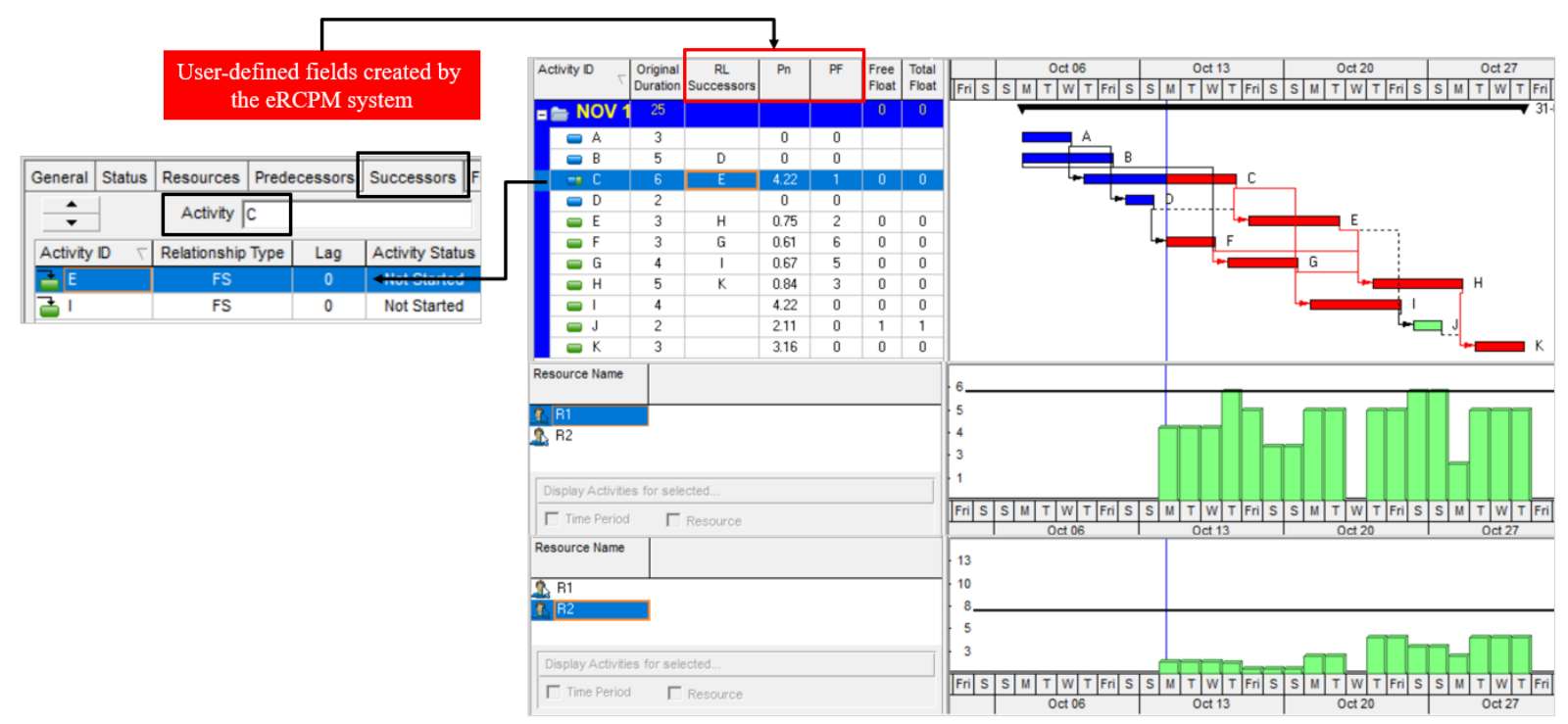

Fig 20. P6 Schedule: Enhanced LF-based Schedule.

The eRCPM also creates a user-defined field called "PF" to display the phantom float values of each activity before adding the resource links into the schedule. P6 users can add another user-defined column called "Pn". This column shows the Priority Number values used by the eRCPM to perform the Enhanced LF heuristic. The Pn values are only added to the P6 file when the default schedule selected by the eRCPM system is the one obtained with this heuristic (Enhanced LF). The PF and Pn values match with the values displayed in Fig.18.

\section{Conclusions}

Traditional Resource-Constrained Scheduling techniques fail to provide correct float values and a continuous critical path in resource-constrained schedules. The lack of resource relationships in a resourceconstrained schedule leads to the calculation of wrong late start/finish dates and the creation of nonexisting floats (phantom float). Therefore, all activities must be considered as influential in the project completion time.

This paper presents the application of the Enhanced Resource Critical Path Method (eRCPM) in a progressed resource-constrained schedule. Primavera P6, a scheduling software frequently used by the construction industry, is not equipped to identify and create resource links when performing an RCS technique. The development of the eRCPM computerized system, which was integrated with Primavera P6, allows the removal of phantom float and the identification of a continuous critical path in P6 resource-constrained schedules.

The eRCPM addresses the fact the activity sequence of a resource-constrained schedule may change after a progress update. The eRCPM system incorporates functionality to keep and remove specific resource relationships of a progressed schedule. This functionally allows the application of the Time Impact Analysis (TIA) methodology for the evaluation of delays. Since this is a contemporaneous analysis, each time a delay is inserted into the schedule specific resource relationships will be kept, removed, and identified.

Additionally, the incorporation of three different heuristics into the eRCPM provides more alternative and flexible schedules that could meet better the project requirements. Moreover, the system selects as default schedule, the one with the shorter duration or with better resource allocation. 


\subsection{Future research and limitations}

Due to the nature of each heuristic, schedulers and project managers should expect to obtain different resource-constrained schedules. The eRCPM performs three different heuristics under a serial approach activities are sorted as a single group and then schedule one at a time. The incorporation of another wellknown RCS method such as the parallel method in the algorithm will provide schedulers more flexibility when selecting the schedule that better meets the project requirements and conditions. Under the parallel approach, the activity sequence is determined and updated at the start of a specific period [11].

Otherwise, the three scenarios defined in the eRCPM to identify resource-driving activities when having several concurrent activities with several predecessor activities were not incorporated in the eRCPM system. Additional work should be carried out to add these criteria to the program.

\section{References}

[1] KPMG. (2017). Make it or Break it. Remaining governance, people, and technology in the construction industry. Global Construction Survey, 2017. S.I: KPMG.

[2] Franco-Duran, D. M., \& de la Garza, J. M. (July 01, 2019). Phantom float in commercial scheduling software. Automation in Construction, 103, 291-299. https://doi.org/10.1016/j.autcon.2019.03.014

[3] Kim, K., and de la Garza, J. M. (2003). Phantom Float. Journal of Construction Engineering and Management, $129,5,507$. https://doi.org/10.1061/40704(2003)48

[4] Franco-Duran, D. M., \& de la Garza, J. M. (November 01, 2019). Review of Resource-Constrained Scheduling Algorithms. Journal of Construction Engineering and Management, 145, 11.). https://doi.org/10.1061/(ASCE)CO.1943-7862.0001698

[5] Wiest, J. D. (1964). Some Properties of Schedules for Large Projects with Limited Resources. Operation Research, 12(3), 395418. https://doi.org/10.1287/opre.12.3.395

[6] Rivera, F. A., \& Duran, A. (January 01, 2004). Critical clouds and critical sets in resource-constrained projects. International Journal of Project Management, 22, 6, 489-497. https://doi.org/10.1016/j.ijproman.2003.11.004

[7] Kim, K. (2003). A Resource-constrained CPM (RCPM) Scheduling and Control Technique with Multiple Calendars. Virginia Tech, Blacksburg, Virginia. Retrieved from https://vtechworks.lib.vt.edu/handle/10919/28139 (Accessed December 6, 2018).

[8] Nisar, S. A., Yamamoto, Koshi., \& Suzuki, K. (January 01, 2013). Resource-dependent Critical Path Method for Identifying the Critical Path and the "Real Floats" in Resource-Constrained Project Scheduling. Construction and Management special Issue / Construction Management Committee, Japan Society of Civil Engineers, 69, 4, 97-107.

[9] Franco-Duran, D. M., \& de la Garza, J. M. (February 12, 2020). Performance of Resource-Constrained Scheduling Heuristics. Journal of Construction Engineering and Management. https://doi.org/10.1061/(ASCE)CO.1943-7862.0001804

[10] Harris, R. B. 1978. Precedence and Arrow Networking Techniques for Construction. Hoboken, NJ: Wiley. ISBN: 978-0-471-04123-8

[11] Moder, J. J., Phillips, C. R., \& Davis, E. W. (1983). Project Management with CPM, PERT, and precedence diagramming (3rd ed. ed.). New York: Van Nostrand Reinhold. ISBN 0960634487- 0442254156 